\title{
CRESCIMENTO DE PLANTAS DE CLONE DE Eucalyptus grandis [HILL ex MAIDEN] SUBMETIDAS A DIFERENTES TRATAMENTOS DE DESRAMA ARTIFICIAL, NA REGIÃO DE CERRADO ${ }^{1}$
}

\author{
Karina Pulrolnik ${ }^{2}$, Geraldo Gonçalves dos Reis ${ }^{3}$, Maria das Graças Ferreira Reis ${ }^{3}$, Marco Antônio Monte ${ }^{4}$ e \\ Ivan da Costa Ilhéu Fontan ${ }^{4}$
}

\begin{abstract}
RESUMO - O crescimento em diâmetro à altura do peito (DAP), altura e volume foi avaliado em plantas de clone de Eucalyptus grandis, submetidas a diferentes intensidades de desrama artificial (altura de 1,0; 1,5; e 2,0 m a partir do solo) e idades de aplicação da primeira intervenção de desrama ( 20 e 28 meses de idade), em Abaeté, MG. A segunda intervenção de desrama em todos os tratamentos foi realizada aos 33 meses de idade, até atingir $3 \mathrm{~m}$ de altura do fuste da planta. Aos 40 meses de idade, observou-se que a desrama artificial não afetou, significativamente ( $p>0,05)$, o crescimento em DAP, altura e volume das plantas submetidas à primeira intervenção de desrama aos 20 meses, porém afetou o DAP e o volume quando a primeira intervenção foi realizada aos 28 meses de idade. Observou-se rápida recuperação da copa das plantas desse clone, independentemente do tratamento de desrama. Considerando não ter havido diferença entre tratamentos para o crescimento das plantas que tiveram a primeira intervenção aos 20 meses de idade e que os galhos vivos ainda apresentavam diâmetro reduzido e praticamente não existiam galhos mortos por ocasião da primeira intervenção nessa idade, recomenda-se a aplicação da desrama nesse clone, na região de cerrado, aproximadamente, aos 20 meses de idade, visando obter madeira de melhor qualidade para serraria.
\end{abstract}

Palavras-chave: Desrama artificial, crescimento e clone de Eucalyptus grandis.

\section{GROWTH OF PRUNED Eucalyptus grandis [HILL ex MAIDEN] CLONES IN THE SAVANNAH REGION, BRAZIL}

\begin{abstract}
The growth in diameter at breast height (DBH), height and volume was evaluated in clones of Eucalyptus grandis [HILL ex MAIDEN] under different artificial pruning intensities $(1.0,1.5$ and $2.0 \mathrm{~m}$ height above ground level) with the first pruning starting at the age of 20 and 28 months. The second pruning for all treatments was performed in plants aged 33 months to obtain a $3 \mathrm{mlog}$. By the age of 40 months, the artificial pruning did not affect significantly $(p>.05)$ tree growth (DHB, height and volume) when the first pruning was applied at the age of 20 months. Pruning starting at 28 months affected significantly DBH and volume growth. A rapid crown recovery was observed for this clone after pruning, independently of the pruning treatment. Taking into account that there was no significant difference in growth when pruning started at 20 months, and that at this age the live branch diameter was still reduced and the number of dead branches was quite low, it is recommended that pruning for this clone established in the Brazilian savannah should be done approximately at the age of 20 months in order to attain high quality boards .
\end{abstract}

Keywords: Artificial pruning, growth, Eucalyptus grandis clone.

\footnotetext{
${ }^{1}$ Recebido em $1^{\circ} .09 .2003$ e aceito para publicação em 20.04.2005.

${ }^{2}$ Programa de Pós-Graduação em Ciência Florestal do DEF/UFV. E-mail: <karinapulrolnik @ bol.com.br>.

${ }^{3}$ Departamento de Engenharia Florestal da Universidade Federal de Viçosa, 36570-000 Viçosa-MG. E-mail: < greis@ ufv.br> e $<$ mgfreis@ufv.br>. Bolsistas do CNPq.

${ }^{4}$ Bolsistas de Iniciação Científica CNPq/UFV.
} 


\section{INTRODUÇÃO}

As empresas florestais têm manejado suas florestas plantadas na busca de agregar qualidade à madeira para atender a um mercado cada vez mais exigente, e o eucalipto tem sido uma alternativa para a produção de madeira serrada de qualidade (PONCE, 1995; NAHUZ et al., 1999). Porém, para a obtenção de madeira de qualidade para serraria, as florestas devem ser manejadas através, principalmente, da aplicação de desrama artificial (PINKARD et al., 1999; PIRES, 2000) e desbaste.

Muitos estudos sobre desrama artificial vêm sendo desenvolvidos para coníferas, conforme revisto por Pires (2000), as quais têm sido tradicionalmente utilizadas para serraria. Recentemente, aumentou o interesse em manejar povoamentos de eucaliptos para uso múltiplo, e algumas espécies desse gênero receberam atenção especial em estudos sobre desrama artificial (BREDENKAMP et al., 1980; PINKARD e BEADLE, 1998; PINKARD et al., 1998; PIRES, 2000; VALE, 2000; PINKARD, 2002).

A desrama artificial pode afetar o crescimento das plantas (BREDENKAMP et al., 1980; PIRES, 2000; PINKARD et al., 1998; PINKARD, 2002). O crescimento da parte aérea e das raízes das plantas está na dependência de um adequado suprimento de reservas orgânicas e inorgânicas, e a remoção de parte da superfície fotossintetizante da planta, através da desrama de galhos vivos, pode promover diminuição drástica dessas reservas (KOZLOWSKI e PALLARDY, 1997), vindo a comprometer o crescimento do tronco das árvores. Harris (1992) comentou que a remoção de galhos em plantas jovens, além de reduzir o suprimento de reservas orgânicas para as raízes, pode diminuir o balanço de reguladores de crescimento na planta. Pires (2000) observou que a desrama de 12,$5 ; 25 ; 50$; e $75 \%$ da altura da copa viva de $E$. grandis promoveu redução substancial no crescimento em altura e diâmetro das plantas, em Dionísio, MG, certamente em conseqüência de uma elevada remoção de área foliar e baixa capacidade de recuperação da copa pelas plantas. No entanto, o crescimento de Eucalyptus nitens não foi comprometido com a desrama de até $50 \%$ da altura da copa viva, o que corresponde a 55\% da área foliar (PINKARD e BEADLE, 1998). Essas variações nas respostas das plantas à aplicação de desrama artificial dependem certamente da capacidade de recuperação da copa das plantas de cada material genético após a desrama, bem como da proporção de área foliar total removida, pois Pires (2000) verificou que $50 \%$ da copa viva correspondia a $80 \%$ da área foliar total.

Segundo Hard (1992), a desrama artificial pode trazer alguns benefícios ao povoamento, permitindo entrada de luz na copa, o que aumentou a fixação de carbono pelas folhas remanescentes. Ainda, segundo Pires (2000), em regiões com acentuado défice hídrico a desrama artificial pode beneficiar o crescimento das plantas, uma vez que a remoção dos galhos basais da copa, que apresentam reduzida capacidade de fixação de carbono, pode também diminuir sua superfície de transpiração.

As dúvidas sobre a desrama artificial em eucalipto referem-se à estação do ano, idade, intensidade e freqüência de aplicação dessa técnica. Conforme Shigo (1989), a desrama deve ser realizada no verão, quando o câmbio está ativo, enquanto Smith (1962) aconselha a primavera e o verão como épocas adequadas para a desrama. A idade da planta em que se realiza a desrama artificial e a intensidade de desrama utilizada são muito variáveis. Vale (2000) aplicou desrama de 0, 2, 4 e 6 m de altura a partir do solo, em plantas de eucalipto aos três anos de idade, ao passo que, no estudo de Pires (2000), a desrama foi aplicada aos 11 meses de idade, utilizando remoção de $0 ; 12,5 ; 25 ; 50 ;$ e 75\% de altura da copa viva. Para Zobel e Buijtenen (1989), a desrama deve ser iniciada quando a árvore estiver suficientemente desenvolvida, e a remoção de galhos vivos nunca deve ser superior à metade da copa. Pinkard et al. (1998) relataram que a desrama deve ser realizada por ocasião do fechamento do dossel, facilitando a entrada de luz através da copa das árvores.

Aparentemente, a intensidade, freqüência e idade de aplicação da desrama artificial variam com o material genético, qualidade do sítio, vigor e idade das plantas, dentre outros. Dessa forma, uma análise detalhada da dinâmica de crescimento da planta pode auxiliar a definição adequada da desrama artificial.

O objetivo do presente estudo foi determinar a capacidade de crescimento das plantas de um clone de Eucalyptus grandis, em resposta à aplicação de diferentes intensidades e idades de aplicação de desrama, em Abaeté, MG. 


\section{MATERIAL E MÉTODOS}

O presente estudo foi desenvolvido em área estabelecida com clone de Eucalyptus grandis [Hill ex Maiden] a partir de 20 meses de idade, estabelecido no espaçamento de $3 \times 3 \mathrm{~m}$, na Companhia Agrícola e Florestal Santa Bárbara Ltda. (CAF), no município de Abaeté, MG, localizado na latitude de 19¹5'94" S e longitude de 45\%44'56" E. A região de estudo apresenta clima subtropical úmido, com temperatura média anual de $22,3{ }^{\circ} \mathrm{C}$, sendo a temperatura média do mês mais frio de $19,0^{\circ} \mathrm{C}$ e, a do mês mais quente, de $23,8^{\circ} \mathrm{C}$. A precipitação média anual é de $1.380 \mathrm{~mm}$. O défice hídrico médio é de $168 \mathrm{~mm}$ anuais e a evapotranspiração potencial anual, de $1.078 \mathrm{~mm}$ (MOREIRA, 1997).

O experimento foi constituído de seis tratamentos, com três repetições, combinando diferentes intensidades de desrama (altura de remoção dos galhos a partir do solo) e proporção de plantas que sofreram desrama (todas as árvores do povoamento ou apenas árvores de qualidade de fuste superior), e a primeira intervenção de desrama foi aplicada em duas idades das plantas, conforme detalhado no Quadro 1.

Nos tratamentos de desrama até a altura de 1,0 e 1,5 $\mathrm{m}$ foram adotadas duas metodologias de remoção de galhos: nos tratamentos 2 e 3 foram removidos galhos até a altura predeterminada (1,0 ou 1,5 m), com a retirada de alguns galhos grossos acima dessa altura, sendo a desrama realizada apenas em árvores com qualidade de fuste elevado, enquanto nos tratamentos 5 e 6 foi feita a remoção de galhos apenas até a altura predeterminada, em todas as árvores do povoamento, independentemente da qualidade do fuste. $\mathrm{O}$ tratamento 4 (remoção de todos os galhos até a altura de $2,0 \mathrm{~m}$ e de alguns galhos grossos acima dessa altura, em árvores selecionadas) foi incluído mesmo considerandose a possibilidade de elevada remoção de área foliar, uma vez que o clone estudado apresentava, com freqüência, galho bastante grosso até a altura de $0,5 \mathrm{~m}$ e vários galhos com diâmetro intermediário entre 1,0 e 1,5 m, cuja remoção implica elevada redução da área foliar na idade em que foi iniciado o experimento.

Nos tratamentos 2, 3 e 4 (Quadro 1) foram desramadas, respectivamente, 56, 65 e 57\% das árvores da parcela dos tratamentos em que a primeira intervenção de desrama foi realizada aos 20 meses e 71,70 e $53 \%$ na primeira intervenção, aos 28 meses. Essas árvores foram selecionadas com base nas características de crescimento, tortuosidade e bifurcação, dentre outros, de modo que fosse possível produzir pelo menos duas toras de $3 \mathrm{~m}$ de comprimento livre de nós.

As duas idades para aplicação da primeira intervenção de desrama foram 20 meses (junho/2000) e 28 meses (agosto/2001); a segunda intervenção de desrama ocorreu aos 33 meses de idade, em todos os tratamentos de desrama, completando $3 \mathrm{~m}$ de tronco desramado (Quadro 1).

A altura média de inserção do primeiro galho vivo, por ocasião da primeira intervenção de desrama, foi de $0,13 \mathrm{~m}$ nas plantas com 20 meses de idade e de $0,46 \mathrm{~m}$ naquelas com 28 meses.

Quadro 1 - Tratamentos de desrama aplicados em plantas de clone de Eucalyptus grandis, com a primeira intervenção aos 20 meses (julho de 2000) e aos 28 meses de idade (março de 2001), em Abaeté, MG

Table 1 - Pruning treatments (intensity and age of the first pruning) applied to Eucalyptus grandis clones in Abaeté, MG, Brazil

\begin{tabular}{cccc}
\hline Tratamentos & \multicolumn{2}{c}{ Altura da Desrama a Partir do Solo (m) } \\
\cline { 2 - 4 } & Primeira Intervenção & $\begin{array}{c}\text { Segunda Intervenção } \\
\text { Altura Máxima de } \\
\text { Desrama (m) }\end{array}$ \\
\hline 1 & 20 e 28 meses & 33 meses & Testemunha \\
2 & $1,0^{1}(+\mathrm{gg})$ & 2,0 & 3,0 \\
3 & $1,5^{1}(+\mathrm{gg})$ & 1,5 & 3,0 \\
4 & $2,0^{1}(+\mathrm{gg})$ & 2,0 & 3,0 \\
6 & $1,0^{2}$ & 1,5 & 3,0 \\
\hline
\end{tabular}

${ }^{1}$ Foram desramadas somente as árvores selecionadas que apresentavam qualidade potencial para uso em serraria, sendo também eliminados alguns galhos grossos (+gg) localizados acima da altura preestabelecida de desrama.

${ }^{2}$ Todas as árvores da parcela foram desramadas até a altura especificada. 
Cada parcela foi constituída de quatro fileiras de 12 plantas, com bordadura simples, ou seja, a área útil de cada parcela foi constituída de duas linhas centrais contendo 10 árvores cada. O experimento foi desenvolvido seguindo o esquema de parcelas subdivididas, tendo nas parcelas os tratamentos e, nas subparcelas, as idades de avaliação, no delineamento inteiramente casualizado, com três repetições.

Após a demarcação das parcelas, antes da aplicação da primeira desrama, foram feitas medições de diâmetro à altura do peito (DAP), altura total da árvore e altura de inserção do primeiro galho vivo, nas plantas da área útil das parcelas (duas linhas de 10 plantas cada). As medições de altura e diâmetro foram realizadas aos 20, 23, 28, 33, 35 e 40 meses de idade.

O volume com casca de madeira por árvore, em $\mathrm{m}^{3}$, foi calculado através do método de Smalian, em que se utilizaram os diâmetros medidos nas alturas de 0,$30 ; 1,30 ; 2,30$; e 3,30 m da planta, aos 23, 28, 33, 35 e 40 meses de idade, respectivamente. Dessa forma, o volume da planta foi determinado até a altura de 3,30 m pelo volume das secções intermediárias através de Smalian, sendo o restante da árvore (acima de 3,30 m de altura) considerado um cone. O volume total das árvores foi obtido pelo somatório do volume das secções intermediárias, apresentadas pelas expressões abaixo:

$$
\begin{aligned}
& \mathrm{V}=\mathrm{V}_{\mathrm{i}}+\mathrm{V}_{\mathrm{c}} \\
& \mathrm{Vi}=\left(\mathrm{g}_{\mathrm{i}}+\mathrm{g}_{\mathrm{i}+1}\right) / 2 * 1_{\mathrm{i}} \\
& \mathrm{Vc}=1 / 3 \mathrm{~g}_{\mathrm{i}} * 1_{\mathrm{i}}
\end{aligned}
$$

em que:

$\mathrm{V}=$ volume final;

$\mathrm{V}_{\mathrm{i}}=$ volume das secções intermediárias;

$\mathrm{V}_{\mathrm{c}=}$ volume do cone;

$\mathrm{g}_{\mathrm{i}=}$ área basal na secção "i";

$\mathrm{g}_{\mathrm{i}+1=}$ área basal na secção “ $\mathrm{i}+1$ ”; $\mathrm{e}$

$1_{i}=$ comprimento da secção.

Os incrementos periódicos de diâmetro, altura e volume foram analisados de modo a identificar as respostas da planta imediatamente após a intervenção da desrama.

Os dados foram analisados por meio de análises de variância e de regressão. Para o fator qualitativo, as médias foram comparadas utilizando o teste de Tukey a 5\% de probabilidade. Para o fator quantitativo, os modelos foram escolhidos baseados na significância dos coeficientes de regressão, empregando-se o teste de $\mathrm{T}$ a $5 \%$ de probabilidade, no coeficiente de determinação, independentemente de ser significativo ou não. Após o ajuste das equações de crescimento em DAP e altura, em cada tratamento foi utilizada a técnica de identidade de modelos.

\section{RESULTADOS E DISCUSSÃO}

\subsection{Crescimento em diâmetro}

Nas plantas que tiveram a primeira intervenção de desrama aos 20 meses de idade (julho/2000) não foram observadas diferenças significativas no DAP das árvores, nas diferentes idades de medição até a idade de 40 meses, no nível de 5\% de probabilidade, pelo teste de Tukey (Quadro 2). Apesar de não ter sido observada diferença estatística, houve ligeira tendência de maior crescimento em diâmetro no tratamento em que a primeira intervenção incluiu a remoção de todos os galhos até a altura de $2,0 \mathrm{~m}$ e de alguns galhos grossos acima desse ponto, que constitui o tratamento de maior intensidade de desrama. Os resultados do presente trabalho contrastam parcialmente com os obtidos por Pires (2000), que observou, aos sete anos de idade, que a remoção de 12,$5 ; 25 ; 50 ;$ e $75 \%$ de altura da copa viva, em $E$. grandis, reduziu significativamente o crescimento em diâmetro e altura das plantas desramadas, proporcionalmente à intensidade de desrama, especialmente com remoção superior a $25 \%$ da altura da copa viva. Vale salientar que essas diferenças de crescimento observadas por Pires (2000) tornaram-se mais evidentes cerca de dois anos após a aplicação da desrama. É provável que em idades mais avançadas ainda possam surgir diferenças significativas no crescimento em diâmetro entre os tratamentos utilizados no presente estudo. De acordo com Smith (1962) e Pires (2000), o crescimento em altura e DAP pode reduzir-se em razão da remoção intensa de galhos vivos da copa da árvore e, conseqüentemente, da área foliar.

Para plantas que tiveram a primeira intervenção de desrama aos 28 meses de idade (março/2001), constatouse que o crescimento em diâmetro diferiu a $5 \%$ de probabilidade, em todos os períodos de medição efetuados, em função da aplicação de desrama (Quadro 2). No tratamento em que houve remoção de galhos até a altura de 1,5 m na primeira intervenção, com remoção de galhos grossos acima desse ponto, foi verificado maior crescimento em diâmetro, porém, desde o início do experimento, as plantas desse tratamento apresentavam crescimento em diâmetro superior ao das plantas dos demais tratamentos, e essa superioridade persistiu durante todo o período experimental. 
Quadro 2 - Médias de diâmetro à altura do peito $(\mathrm{cm})$, em diferentes idades, de plantas de clone de Eucalyptus grandis submetidas à primeira intervenção de desrama aos 20 e 28 meses de idade, em Abaeté, MG

Table 2 - Mean diameter (cm) of Eucalyptus grandis clone submitted to the first pruning at the age of 20 and 28 months, in Abaeté, MG, Brazil

\begin{tabular}{|c|c|c|c|c|c|c|}
\hline \multirow{2}{*}{$\begin{array}{c}\text { Altura de } \\
\text { Desrama }(\mathrm{m})^{1}\end{array}$} & \multicolumn{5}{|c|}{ Idade (meses) } & \multirow[b]{2}{*}{40} \\
\hline & 20 & 23 & 28 & 33 & 35 & \\
\hline \multicolumn{7}{|c|}{ Primeira intervenção aos 20 meses } \\
\hline Testemunha & $4,60 \mathrm{a}$ & $5,00 \mathrm{a}$ & 6,73 a & 8,06 a & 8,26 a & 9,89 a \\
\hline $1,0(+g g)+2,0$ & $4,50^{2}$ & $4,55 \mathrm{a}$ & 6,83 a & $8,10 \mathrm{a}$ & 8,23 a & 9,60 a \\
\hline $1,5(+g g)+1,5$ & $5,00^{2}$ & 4,91 a & 7,27 a & 8,10 a & 8,29 a & 9,81 a \\
\hline $2,0(+g g)+1,0$ & $5,24^{2}$ & 5,10 a & 7,28 a & 8,35 a & 8,56 a & $10,20 \mathrm{a}$ \\
\hline $1,0+2,0$ & $4,56 \mathrm{a}$ & 4,73 a & 6,39 a & 7,47 a & $7,60 \mathrm{a}$ & 9,40 a \\
\hline $1,5+1,5$ & $4,63 \mathrm{a}$ & 5,08 a & 6,78 a & 7,87 a & 8,03 a & 9,45 a \\
\hline \multicolumn{7}{|c|}{ Primeira intervenção aos 28 meses } \\
\hline Testemunha & -- & -- & $6,73 \mathrm{ab}$ & $8,06 \mathrm{ab}$ & $8,26 \mathrm{ab}$ & $9,89 \mathrm{ab}$ \\
\hline $1,0(+g g)+2,0$ & -- & -- & $6,87 \mathrm{ab}$ & $7,83 \mathrm{ab}$ & $8,10 \mathrm{ab}$ & $9,51 \mathrm{ab}$ \\
\hline $1,5(+g g)+1,5$ & -- & -- & $7,57 \mathrm{a}$ & 8,48 a & 8,69 a & $10,00 \mathrm{a}$ \\
\hline $2,0(+g g)+1,0$ & -- & -- & $6,78 \mathrm{ab}$ & $7,27 \quad b$ & $7,42 \quad b$ & $9,23 \mathrm{ab}$ \\
\hline $1,0+2,0$ & -- & -- & $6,21 \mathrm{~b}$ & $7,17 \quad b$ & $7,20 \quad b$ & $8,81 \quad b$ \\
\hline $1,5+1,5$ & -- & -- & $5,93 \mathrm{~b}$ & $7,55 \mathrm{ab}$ & $7,59 \mathrm{ab}$ & $9,24 \mathrm{ab}$ \\
\hline
\end{tabular}

Médias seguidas da mesma letra minúscula na coluna, para cada idade de intervenção, não diferem entre si, pelo teste de Tukey a 5\%. ${ }^{1}+\mathrm{gg}=$ remoção de alguns galhos acima da altura definida do fuste limpo. Por exemplo, o tratamento $1,0(+\mathrm{gg})+2,0$ significa desrama até $1,0 \mathrm{~m}$ e remoção de alguns galhos grossos (+gg) acima dessa altura aos 20 meses e remoção de galhos entre 1 e 3 m de altura da planta, aos 33 meses, totalizando $3 \mathrm{~m}$ de altura de desrama.

${ }^{2}$ Dados provenientes de uma repetição.

O teste de identidade de modelos possibilitou a adoção de uma única equação para todos os tratamentos, em substituição àquelas obtidas em cada tratamento de desrama, para as plantas que tiveram a primeira intervenção de desrama aos 20 meses de idade, em razão de não ter sido observado diferença significativa entre elas. Nas plantas que sofreram a primeira intervenção de desrama aos 28 meses, verificou-se, através de teste de identidade de modelos, que as equações devem ser usadas separadamente em todos os tratamentos (Quadro 3).
As plantas dos tratamentos 1,0 (+gg)+2,0 e 1,0+2,0, que tiveram a primeira intervenção de desrama aos 20 meses de idade, apresentaram incremento periódico em diâmetro mais elevado (Figura 1) do que os demais tratamentos, nos três meses subseqüentes à desrama, possivelmente em razão de possuírem maior proporção de copa remanescente. Nos demais tratamentos houve a recuperação da copa para, posteriormente, haver o crescimento em diâmetro. No período entre outubro e fevereiro, o que corresponde à época de maior pluviosidade na região, os tratamentos submetidos

Quadro 3 - Equações para estimar o diâmetro à altura do peito (DAP), em cm, de plantas de clone de Eucalyptus grandis submetidas à primeira intervenção de desrama aos 20 e 28 meses de idade, em função da idade (I), em meses, em Abaeté, MG

Table 3 - Regression equations to estimate diameter $(\mathrm{cm})$ of Eucalyptus grandis clone with the first pruning done at the age of 20 and 28 months, in Abaeté, MG, Brazil

\begin{tabular}{lcc}
\hline Altura de Desrama $(\mathrm{m})$ & Equação & $\mathrm{R}^{2}$ Ajustado \\
\hline \multirow{2}{*}{ Equação geral } & Primeira intervenção aos 20 meses \\
& Ln DAP $=3,1631-35,9720 *(1 / \mathrm{I})$ & 0,86 \\
Testemunha & Primeira intervenção aos 28 meses \\
$1,0(+\mathrm{gg})+2,0$ & Ln DAP $=3,1421-34,9521 *(1 / \mathrm{I})$ & 0,78 \\
$1,5(+\mathrm{gg})+1,5$ & Ln DAP $=2,9588-29,3389 *(1 / \mathrm{I})$ & 0,87 \\
$2,0(+\mathrm{gg})+1,0$ & Ln DAP $=2,9093-25,2033 *(1 / \mathrm{I})$ & 0,70 \\
$1,0+2,0$ & Ln DAP $=2,8349-26,8886 *(1 / \mathrm{I})$ & 0,75 \\
$1,5+1,5$ & Ln DAP $=2,9144-31,0315 *(1 / \mathrm{I})$ & 0,91 \\
\hline
\end{tabular}


à maior intensidade de desrama apresentaram aumento substancial do incremento periódico em razão da recomposição da copa no período anterior, mantendo o crescimento em diâmetro dessas plantas. A capacidade de retomada rápida de crescimento após a desrama artificial foi observada em Eucalyptus grandis, em Zululand (África), por Bredenkamp et al. (1980), com a remoção de galhos correspondente a valores inferiores a 50\% da altura da copa viva. Pinkard e Beadle (1998) observaram que, dois anos após a desrama artificial, houve recuperação da proporção da copa viva removida até $50 \%$ de altura da copa viva (55\% da área foliar), em Eucalyptus nitens. Ou seja, é possível aplicar desrama artificial em povoamentos de eucalipto visando à melhoria da qualidade da madeira, sem prejuízo para o crescimento das plantas, quando estas têm capacidade de recuperação rápida de sua copa.

Entre 23 (outubro/2000) e 28 meses (março/2001), primeira estação chuvosa após a primeira intervenção de desrama, foi verificado que as plantas de todos os tratamentos apresentaram incremento periódico em diâmetro similar, exceto no tratamento 1,0+2,0, que teve redução de incremento (Figura 1). Essa similaridade no crescimento das plantas dos diversos tratamentos possivelmente se deve, também, ao efeito da liberação intensa de nutrientes das folhas verdes caídas no solo com a desrama, pois, conforme revisado por Ferreira (1984), folhas verdes apresentam decomposição mais rápida e, certamente, os nutrientes dos resíduos da desrama foram liberados em grande quantidade, ainda, durante a estação chuvosa, sendo prontamente utilizadas pelas plantas que exibiram crescimento bastante ativo.

Na segunda estação chuvosa (outubro/2001 a março/ 2002), as plantas também apresentaram incremento periódico em diâmetro similar nos diferentes tratamentos, exceto naquele com a desrama do fuste de 1,5+1,5 m. Vale salientar que em agosto/2001 foi realizada a desrama para completar $3 \mathrm{~m}$ de altura em todos os tratamentos, e possivelmente houve efeito positivo do nutriente contido nos resíduos da desrama sobre o crescimento das plantas.

Nas plantas que tiveram a primeira desrama aos 28 meses de idade, observou-se redução substancial no incremento periódico em diâmetro, no tratamento 2,0(+gg)+1,0 (Figura 1). Porém, no último período de avaliação, que coincidiu com o período chuvoso, as plantas desse tratamento recuperaram o crescimento, apresentando elevado incremento periódico. Não foi observado um comprometimento do crescimento em diâmetro das plantas nesse tratamento em razão, possivelmente, da rápida recomposição da copa, bem como do fato de grande parte das folhas removidas aos 28 meses de idade se constituir de folhas em senescência. Também, já havia ocorrido intensa abscisão foliar na parte basal da copa, anterior à remoção dos galhos. As plantas desramadas até $2 \mathrm{~m}$ de altura podem, também, ter sido beneficiadas pelo aumento da incidência de radiação sobre a copa remanescente. (a)

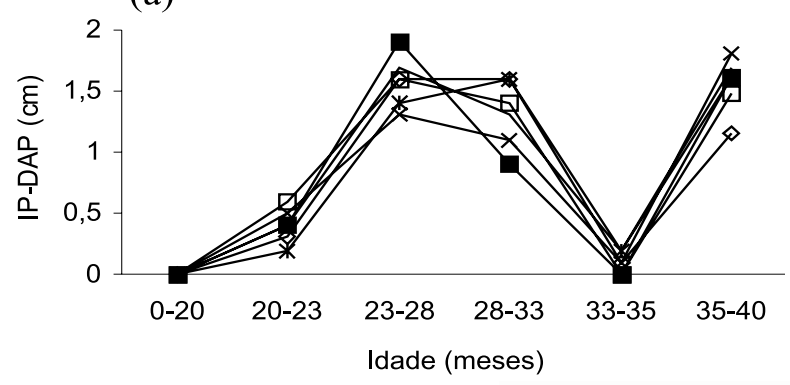

(b)

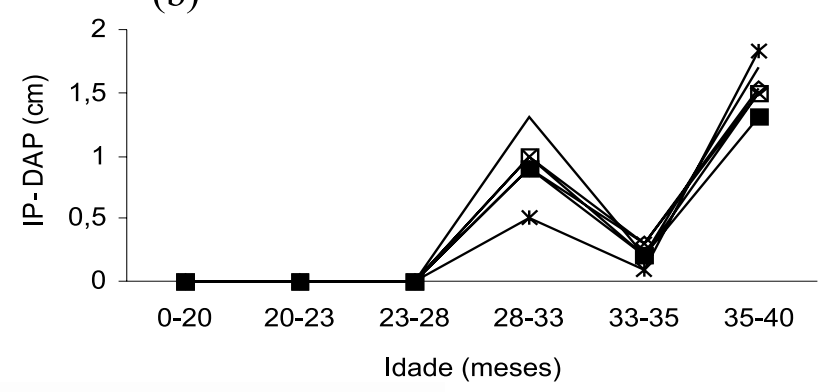

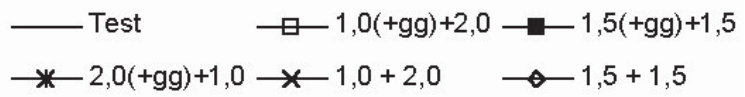

Figura 1 - Incremento periódico do crescimento em diâmetro à altura do peito (IP-DAP), em cm, de plantas de clone de Eucalyptus grandis submetidas à primeira intervenção de desrama aos 20 (a) e 28 (b) meses de idade, em função da idade (meses), em Abaeté, MG.

Figure 1 - Periodic increment of growth in diameter (cm) of Eucalyptus grandis clone plants submitted to the first pruning intervention at the age of 20 and 28 months, in Abaeté, MG, Brazil.

R. Árvore, Viçosa-MG, v.29, n.4, p.495-505, 2005

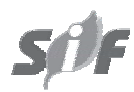


Quando a desrama é feita apenas em parte das árvores do povoamento, a competição das plantas vizinhas não desramadas com as plantas desramadas torna-se acentuada, uma vez que estas últimas perdem parte de sua superfície fotossintetizante efetiva, colocando-as em desvantagem com aquelas que não receberam desrama. É provável que, nesse caso, o primeiro desbaste deve ser realizado o mais rapidamente possível, para evitar prejuízos ao crescimento das plantas desramadas, que apresentam maior valor comercial.

\subsection{Crescimento em altura}

Não foi observado diferença significativa em altura entre os tratamentos em que a primeira intervenção de desrama foi realizada aos 20 meses (Quadro 4). Porém, o crescimento em altura de plantas submetidas à primeira desrama aos 28 meses (março/2001) variou significativamente entre tratamentos aos 28,33 e 35 meses de idade das plantas, com a ressalva de que as diferenças desapareceram na última avaliação, aos 40 meses de idade.

Pelo teste de identidade de modelos, foi constatado que uma única equação pode ser utilizada para estimar a altura das plantas nos seis tratamentos de desrama, quando a primeira intervenção de desrama foi realizada aos 20 meses de idade, enquanto nas plantas que sofreram a primeira intervenção aos 28 meses as equações devem ser usadas separadamente em cada tratamento (Quadro 5).

Quadro 4 - Médias da altura (m), em diferentes idades, de plantas de clone de Eucalyptus grandis submetidas à primeira intervenção de desrama aos 20 e 28 meses de idade, em Abaeté, MG

Table 4 -Mean height $(m)$ of Eucalyptus grandis clone with the first pruning done at the age of 20 and 28 months, in Abaeté, MG, Brazil

\begin{tabular}{|c|c|c|c|c|c|c|}
\hline \multirow{2}{*}{$\begin{array}{c}\text { Altura de } \\
\text { Desrama }(\mathrm{m})^{1}\end{array}$} & \multicolumn{6}{|c|}{ Idade (meses) } \\
\hline & 20 & 23 & 28 & 33 & 35 & 40 \\
\hline \multicolumn{7}{|c|}{ Primeira intervenção aos 20 meses } \\
\hline Test. & $6,40 \mathrm{a}$ & 6,77 a & 8,59 a & $10,70 \mathrm{a}$ & 10,92 a & $12,86 \mathrm{a}$ \\
\hline $1,0(+g g)+2,0$ & $6,43^{1} \mathrm{a}$ & 6,35 a & 8,43 a & $9,67 \mathrm{a}$ & $10,11 \mathrm{a}$ & $12,10 \mathrm{a}$ \\
\hline $1,5(+\mathrm{gg})+1,5$ & $6,54^{1} \mathrm{a}$ & 6,68 a & 8,69 a & $9,77 \mathrm{a}$ & $10,00 \mathrm{a}$ & $12,33 \mathrm{a}$ \\
\hline $2,0(+g g)+1,0$ & $6,80^{1}$ a & 7,04 a & 8,81 a & $9,90 \mathrm{a}$ & $10,28 \mathrm{a}$ & $12,43 \mathrm{a}$ \\
\hline $1,0+2,0$ & 6,50 a & 6,68 a & 8,13 a & $9,57 \mathrm{a}$ & $10,22 \mathrm{a}$ & $12,20 \mathrm{a}$ \\
\hline $1,5+1,5$ & 6,52 a & $6,76 \mathrm{a}$ & 8,41 a & $9,70 \mathrm{a}$ & $10,32 \mathrm{a}$ & $12,40 \mathrm{a}$ \\
\hline \multicolumn{7}{|c|}{ Primeira intervenção aos 28 meses } \\
\hline Test. & - & - & $8,59 \mathrm{ab}$ & $10,71 \mathrm{ab}$ & $10,97 \mathrm{ab}$ & $12,86 \mathrm{a}$ \\
\hline $1,0(+g g)+2,0$ & - & - & $8,70 \mathrm{ab}$ & $9,40 \quad b$ & $10,10 \mathrm{ab}$ & 12,29 a \\
\hline $1,5(+g g)+1,5$ & - & - & 10,08 a & $11,53 \mathrm{a}$ & $11,97 \mathrm{a}$ & $13,40 \mathrm{a}$ \\
\hline $2,0(+g g)+1,0$ & - & - & $8,67 \mathrm{ab}$ & $9,07 \quad b$ & $10,29 \mathrm{ab}$ & $12,21 \mathrm{a}$ \\
\hline $1,0+2,0$ & - & - & 8,12 b & $9,47 \quad b$ & $9,53 \mathrm{~b}$ & $11,87 \mathrm{a}$ \\
\hline $1,5+1,5$ & - & - & $7,67 \quad b$ & $10,23 \mathrm{ab}$ & $10,23 \mathrm{ab}$ & $11,79 \mathrm{a}$ \\
\hline
\end{tabular}

Médias seguidas da mesma letra minúscula na coluna, para cada idade de intervenção, não diferem entre si, pelo teste de Tukey a 5\% de probabilidade.

Quadro 5 - Equações para estimar a altura $(\mathrm{H})$, em metros, de plantas de clone de Eucalyptus grandis submetidas à primeira intervenção de desrama aos 20 e 28 meses de idade, em função da idade (I), em meses, em Abaeté, MG

Table 5 -Regression equations to estimate heigth $(\mathrm{m})$ of Eucalyptus grandis clone plants submitted to the first pruning intervention at the age of 20 and 28 months, in Abaeté, MG, Brazil

\begin{tabular}{lcc}
\hline Altura de Desrama $(\mathrm{m})$ & \multicolumn{1}{c}{ Equação } & $\mathrm{R}^{2}$ Ajustado \\
\hline & Primeira intervenção aos 20 meses & \\
Equação geral & Ln H $=3,2646-31,5735 *(1 / \mathrm{I})$ & \\
& Primeira intervenção aos 28 meses & 0,92 \\
Testemunha & Ln H $=3,4702-36,9823 *(1 / \mathrm{I})$ & 0,75 \\
$1,0+\mathrm{gg}+2,0$ & Ln H $=3,2335-31,0278 *(1 / \mathrm{I})$ & 0,82 \\
$1,5+\mathrm{gg}+1,5$ & Ln H $=3,2358-26,1267 *(1 / \mathrm{I})$ & 0,54 \\
$2,0+\mathrm{gg}+1,0$ & Ln H $=3,2457-31,6652 *(1 / \mathrm{I})$ & 0,79 \\
$1,0+2,0$ & Ln H $=3,2737-33,6710 *(1 / \mathrm{I})$ & 0,79 \\
$1,5+1,5$ & Ln H $=3,5081-40,8980 *(1 / \mathrm{I})$ & \\
\hline
\end{tabular}


Entre 28 e 33 meses de idade, o incremento periódico em altura foi mais elevado nas plantas da testemunha, enquanto todos os tratamentos que sofreram desrama apresentaram redução no incremento periódico nesse período (Figura 2). Entre 35 e 40 meses, o incremento periódico das plantas do tratamento $1,5(+\mathrm{gg})+1,5$ foi o mais baixo, ao passo que nos demais tratamentos os incrementos foram similares. Nas plantas que tiveram a primeira intervenção de desrama aos 28 meses de idade, a testemunha também manteve incremento periódico em altura mais elevado, enquanto as plantas dos tratamentos com maior altura de remoção de galhos apresentaram, inicialmente, incremento periódico reduzido, havendo, porém, recuperação posterior.

Com base nos resultados de crescimento em diâmetro e altura aqui apresentados, verificou-se que as intervenções de desrama realizadas não tiveram efeitos negativos significativos sobre o crescimento das plantas do clone estudado até a idade de 40 meses, na região de Abaeté, MG, principalmente quando a desrama foi feita em plantas mais jovens. Segundo Young e Kramer (1954), citados por Kramer e Kozlowski (1972), o crescimento em altura se suporta, principalmente, pela ação de reguladores de crescimento e de hidratos de carbono produzidos na copa da árvore, nas proximidades do ápice. Assim, quando acontece uma desrama, parte da copa localizada em estratos inferiores, sobretudo com eliminação de folhas em senescência da copa das árvores, pode não comprometer substancialmente a

(a)

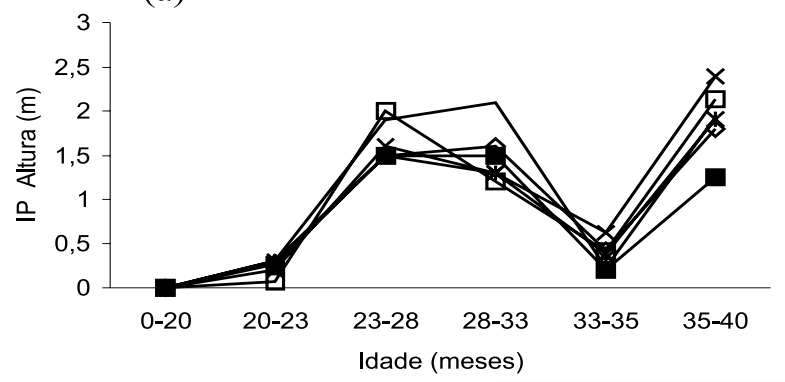

produção de hidratos de carbono, que são utilizados em favor do alongamento da árvore, e, assim, manter o desenvolvimento desta, tanto em altura quanto em diâmetro. Também, devem-se levar em conta, na análise desses resultados, a pronta recuperação da copa das plantas desramadas desse clone e a eliminação de fortes drenos, em razão da eliminação de galhos grossos que se localizam até a altura de aproximadamente 1,5 m de altura.

\subsection{Produção volumétrica}

A produção volumétrica integra os crescimentos em altura e em diâmetro da planta, ambos não afetados significativamente pelos tratamentos de desrama, especialmente nas plantas que tiveram a primeira intervenção de desrama aos 20 meses de idade, conforme discutido anteriormente. Nas plantas que sofreram a primeira intervenção aos 28 meses, a produção volumétrica apresentou variação significativa entre os tratamentos (Quadro 6). A maior produção volumétrica foi obtida com a aplicação do tratamento 1,5(+gg)+1,5, seguido da testemunha. Nessa idade, as plantas já apresentavam alguma abscisão foliar, não tendo ocorrido elevada remoção de folhas com a desrama. Observouse que a remoção de alguns galhos grossos entre 1 e $2 \mathrm{~m}$ de altura foi benéfica para o crescimento das plantas, pois aquelas dos tratamentos com remoção dos galhos apenas até a altura de 1,0 ou 1,5 m apresentaram menor volume.

(b)

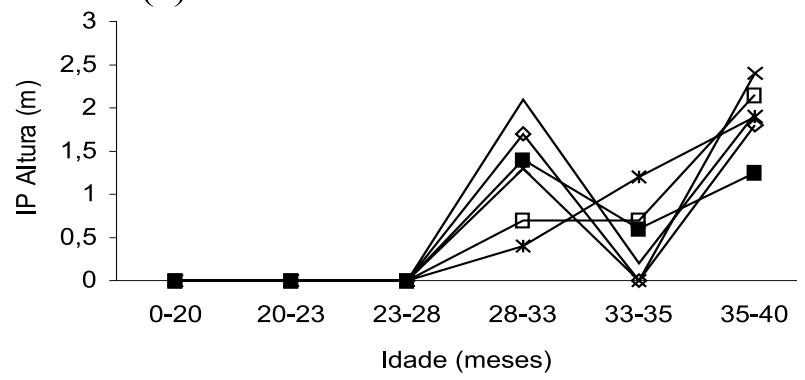

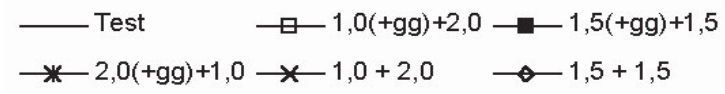

Figura 2 - Incremento periódico do crescimento em altura (IP-Altura), em metros, de plantas de clone de Eucalyptus grandis submetidas à primeira intervenção de desrama aos 20 (a) e 28 (b) meses de idade, em função da idade (meses), em Abaeté, MG.

Figure 2 - Periodic increment of growth in heigth $(m)$ of Eucalyptus grandis clone plants submitted to the first pruning intervention at the age of 20 and 28 months, in Abaeté, MG, Brazil.

R. Árvore, Viçosa-MG, v.29, n.4, p.495-505, 2005

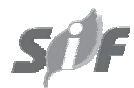


Quadro 6 - Médias do volume ( $\mathrm{m}^{3}$ /árvore), em diferentes idades de plantas de clone de Eucalyptus grandis submetidas à primeira intervenção de desrama aos 20 e 28 meses, em Abaeté, MG

Table 6 -Average volume ( $\mathrm{m}^{3} /$ tree $)$ of Eucalyptus grandis clone plants submitted to the first pruning intervention at the age of 20 and 28 months, in Abaeté, MG, Brazil

\begin{tabular}{|c|c|c|c|c|c|}
\hline \multirow{2}{*}{$\begin{array}{c}\text { Altura de } \\
\text { Desrama (m) }\end{array}$} & \multicolumn{5}{|c|}{ Idade (meses) } \\
\hline & 23 & 28 & 33 & 35 & 40 \\
\hline & \multicolumn{5}{|c|}{ Primeira intervenção aos 20 meses } \\
\hline Testemunha & $0,0074 \mathrm{a}$ & 0,0162 a & 0,0241 a & 0,0276 a & 0,0433 a \\
\hline $1,0(+g g)+2,0$ & 0,0071 a & 0,0158 a & $0,0215 \mathrm{a}$ & $0,0275 \mathrm{a}$ & 0,0397 a \\
\hline $1,5(+\mathrm{gg})+1,5$ & 0,0084 a & 0,0168 a & 0,0219 a & 0,0278 a & 0,0427 a \\
\hline $2,0(+\mathrm{gg})+1,0$ & 0,0088 a & $0,0194 \mathrm{a}$ & 0,0258 a & $0,0292 \mathrm{a}$ & $0,0444 \mathrm{a}$ \\
\hline $1,0+2,0$ & $0,0065 \mathrm{a}$ & 0,0141 a & $0,0205 \mathrm{a}$ & $0,0244 \mathrm{a}$ & $0,0380 \mathrm{a}$ \\
\hline \multirow[t]{2}{*}{$1,5+1,5$} & $0,0074 \mathrm{a}$ & $0,0160 \mathrm{a}$ & $0,0220 \mathrm{a}$ & $0,0240 \mathrm{a}$ & 0,0391 a \\
\hline & \multicolumn{5}{|c|}{ Primeira intervenção aos 28 meses } \\
\hline Testemunha & -- & -- & $0,0241 \mathrm{ab}$ & $0,0276 \mathrm{ab}$ & $0,0433 \mathrm{ab}$ \\
\hline $1,0(+g g)+2,0$ & - & - & $0,0202 \mathrm{ab}$ & $0,0246 \quad b$ & $0,0401 \mathrm{ab}$ \\
\hline $1,5(+\mathrm{gg})+1,5$ & - & - & 0,0296 a & 0,0377 a & $0,0470 \mathrm{a}$ \\
\hline $2,0(+\mathrm{gg})+1,0$ & - & -- & $0,0184 \mathrm{ab}$ & $0,0219 b$ & $0,0399 \mathrm{ab}$ \\
\hline $1,0+2,0$ & - & - & $0,0163 \mathrm{~b}$ & 0,0175 & $0,0336 \quad b$ \\
\hline $1,5+1,5$ & - & - & $0,0197 \mathrm{ab}$ & 0,0199 & $0,0356 \mathrm{ab}$ \\
\hline
\end{tabular}

Médias seguidas da mesma letra minúscula na coluna, para cada idade de intervenção, não diferem entre si, pelo teste de Tukey a 5\% de probabilidade.

As equações de regressão selecionadas para estimar o volume de plantas de clone de Eucalyptus grandis, sob diferentes intensidades de desrama, em função da idade, nas duas idades iniciais de intervenção de desrama (20 e 28 meses, respectivamente), são mostradas no Quadro 7. Constatou-se que a equação Ln V = -1,1226 - 86,5286* (1/I), com $\mathrm{R}^{2}$ ajustado de 0,91 , pode ser usada para estimar o volume das árvores, em função da idade, para plantas que tiveram primeira intervenção de desrama aos 20 meses, independentemente do tratamento, em razão de não ter sido verificado diferença significativa em volume entre os tratamentos. Nas plantas que tiveram a primeira intervenção de desrama aos 28 meses, o teste de identidade de modelos indicou que, em todos os tratamentos, devem-se utilizar as equações individuais.

Nas plantas que tiveram primeira intervenção de desrama aos 20 meses, as diferenças no incremento periódico do volume foram reduzidas, confirmando que a desrama não foi suficientemente drástica a ponto de diminuir o crescimento das plantas, como constatado anteriormente pela análise do crescimento em altura e diâmetro (Figura 3). O incremento periódico em volume entre 35 e 40 meses, nas plantas que tiveram primeira intervenção de desrama aos 28 meses, foi maior no tratamento em que houve a remoção de galhos até 2,0 m de altura na primeira intervenção, com remoção de galhos grossos acima desse ponto, seguido do tratamentotestemunha.

Quadro 7 - Equações para estimar o volume (V), em mª́rvore, de plantas de clone de Eucalyptus grandis submetidas à primeira intervenção de desrama aos 20 e 28 meses de idade, em função da idade (meses), em Abaeté, MG

Table 7 - Regression equations to estimate volume $\left(\mathrm{m}^{3} /\right.$ tree) of Eucalyptus grandis clone plants submitted to the first pruning at the age of 20 and 28 months, in Abaeté, MG, Brazil

\begin{tabular}{llc}
\hline Altura de Desrama $(\mathrm{m})$ & \multicolumn{1}{c}{ Equação } \\
\hline \multirow{2}{*}{ Equação geral } & Primeira intervenção aos 20 meses & $\mathrm{R}^{2}$ Ajustado \\
& Ln V $=-1,1226-86,5286 *(1 / \mathrm{I})$ & \\
Testemunha & Primeira intervenção aos 28 meses & 0,91 \\
$1,0(+\mathrm{gg})+2,0$ & Ln V $=-0,3010-114,4750 *(1 / \mathrm{I})$ & 0,61 \\
$1,5(+\mathrm{gg})+1,5$ & Ln V $=-0,0410-130,7550 *(1 / \mathrm{I})$ & 0,83 \\
$2,0(+\mathrm{gg})+1,0$ & Ln V $=-0,92480-84,9610 *(1 / \mathrm{I})$ & 0,45 \\
$1,0+2,0$ & Ln V $=-0,4680-148,3910^{*}(1 / \mathrm{I})$ & 0,95 \\
$1,5+1,5$ & Ln V $=-0,1610-143,6020 *(1 / \mathrm{I})$ & 0,94 \\
\hline
\end{tabular}


(a)

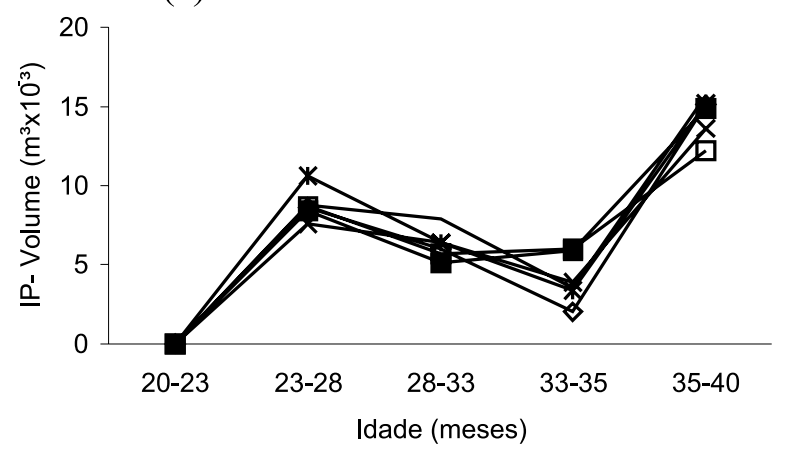

(b)

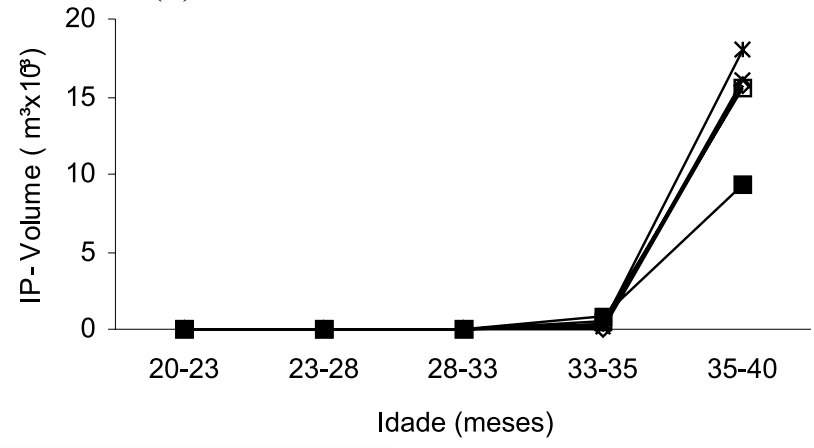

$$
\begin{aligned}
& \text { - Test } \quad \square-1,0(+\mathrm{gg})+2,0 \longrightarrow-1,5(+\mathrm{gg})+1,5 \\
& *-2,0(+g g)+1,0 \rightarrow-1,0+2,0 \multimap 1,5+1,5
\end{aligned}
$$

Figura 3 - Incremento periódico do volume médio (IP-Volume), em mª́rvore, de plantas de clone de Eucalyptus grandis submetidas à primeira intervenção de desrama aos 20 e 28 meses de idade, em função da idade (meses), em Abaeté, MG

Figure 3 - Periodic increment of growth in volume $\left(\mathrm{m}^{3} /\right.$ tree $)$ of Eucalyptus grandis clone plants submitted to the first pruning intervention at the age of 20 and 28 months, in Abaeté, MG, Brazil.

Nas plantas que tiveram primeira intervenção de desrama aos 28 meses, o incremento periódico nas plantas do tratamento $1,5(+\mathrm{gg})+1,5$ foi bem mais elevado que nos demais, mas apresentou redução no último período de avaliação. Nos tratamentos em que não foi realizada a remoção de galhos grossos em maiores alturas, o incremento periódico manteve-se muito baixo imediatamente após a primeira intervenção de desrama. Porém, verificou-se aumento no seu incremento periódico após a segunda intervenção de desrama aos 33 meses de idade, quando foram removidos galhos até a altura de $3 \mathrm{~m}$.

\section{CONCLUSÕES}

De maneira generalizada, os resultados do presente estudo indicam que os tratamentos de desrama artificial aplicados ao clone de Eucalyptus grandis estudado não foram suficientemente drásticos, de modo a comprometer substancialmente o desenvolvimento das plantas até a idade de 40 meses. Considerando que não houve diferença significativa de crescimento entre tratamentos na primeira época de desrama, que os galhos vivos ainda apresentam diâmetro reduzido e que praticamente não existem galhos mortos por ocasião da primeira intervenção, recomenda-se a aplicação da desrama nesse clone por volta dos 20 meses de idade, visando obter madeira de melhor qualidade para serraria.

\section{AGRADECIMENTOS}

À Coordenação de Aperfeiçoamento de Pessoal de Ensino Superior (CAPES), pela bolsa de estudos; e ao CNPq, à FAPEMIG e à Companhia Agrícola e Florestal Santa Bárbara Ltda., pelo apoio técnico e financeiro.

\section{REFERÊNCIAS BIBLIOGRÁFICAS}

BREDENKAMP, B.V.; MALAN, F.S.; CONRADIE, W.E. Some effects of pruning on growth and timber quality of Eucalyptus grandis in Zululand. South African Forestry Journal, v.114, p.29-34, 1980.

FERREIRA, M.G.M. An analysis of the productivity of Eucalyptus grandis plantations in the "Cerrado" region in Brazil: a nutrient cycling approach. Vancouver: The University of Brithish Columbia, 1984. 230p.

HARD, J. Success of spruce beetle attacks in pruned and unpruned boles of Lutz spruce in south-central Alaska. Forest Ecology and Managament, v.47, p.51-70, 1992. 
HARRIS, R.W. Arboriculture: integrated management of landscape trees, shrubs, and vines. 2. ed. New Jersey: Prentice-Hall, 1992.674 p.

KOZLOWSKI, T.T.; PALLARDY, S.G

Physiology of woody plants. San Diego: Academic Press, 1997. v.2.411p.

KRAMER, P.J.; KOZLOWSKI, T.T. Fisiologia das árvores. Lisboa: Fundação Calouste Gulbenkian, 1972.745 p.

MOREIRA, I.P.S. Classificação ecológica do território brasileiro situado entre 16 e $^{2} 4^{\circ}$ latitude sul e 48 e $39^{\circ} 51^{\prime}$ longitude oeste. 1997. 156f. Tese (Doutorado em Ciência Florestal) - Universidade Federal de Viçosa, Viçosa, 1997.

NAHUZ, M.A.R.; MIRANDA, M.J.A.; FRANCO, N. Inovações na área de utilização da madeira de eucalipto: a fabricação de móveis. In: WORKSHOP SOBRE TÉCNICAS DE ABATE, PROCESSAMENTO E UTILIZAÇÃO DA MADEIRA DE EUCALIPTO, 1999, Viçosa Anais... Viçosa: SIF, 1999. p.28-30.

PINKARD, E.A.; BEADLE, C. L. Effects of green pruning on growth and stem shape of Eucalyptus nitens (Deane and Maiden) Maiden. New Forests, v.15, p.107-126, 1998.

PINKARD, E.A.; BEADLE, C.L. Above-ground biomass partitioning and crown architecture of Eucalyptus nitens (Deane and Maiden) Maiden following green pruning. Canadian Journal of Forest Research, v.28, p.1419-1428, 1999.
PINKARD, E.A. et al. Modelling the effect of physiological responses to green pruning on net biomass production of Eucalyptus nitens (Deane and Maiden) Maiden. Tree Physiology, v.19, p.1-12, 1998.

PINKARD, E. Effects of pattern and severity of pruning on growth and branch development of pre-canopy closure Eucalyptus nitens. Forest Ecology and Management, v. 157, p.127230, 2002.

PIRES, B.M. Efeito da desrama artificial no crescimento e qualidade da madeira de Eucalyptus grandis para serraria. 2000. 96p. Dissertação (Mestrado em Ciência Florestal) - Universidade Federal de Viçosa, Viçosa, 2000.

PONCE, R.H. Eucalipto como madeira serrada: desafios e perspectivas. In: SEMINÁRIO INTERNACIONAL DE UTILIZAÇÃO DA MADEIRA DE EUCALIPTO PARA SERRARIA, São Paulo. 1995. Anais... Piracicaba: IPEF/IPT, 1995. p.51-60.

SHIGO, A.L. Tree pruning: a world photo guide. Durham: Shigo and Trees Associates, 1989.

SMITH, D.M. The practice of silviculture. New York: John Wiley \& Sons, 1962. 578 p.

VALE, R.S. Efeito da desrama artificial no crescimento e na qualidade da madeira de clones de eucalipto, em sistema agro-silvo-pastoril. 2000. $96 \mathrm{f}$. Dissertação (Mestrado em Engenharia Florestal) Universidade Federal de Lavras, Lavras, 2000.

ZOBEL, B.J.; VAN BUIJTENEN J.P. Wood variation: Its causes and control. New York: Springer Verlag, 1989. 363 p. 